\title{
Atelosteogenesis Type 2/Diastrophic Dysplasia Phenotypic Spectrum: From Prenatal to Preimplantation Genetic Diagnosis
}

\author{
Eduardo P. Mattos',2, José Antônio A. Magalhães ${ }^{3}$, Lauréane Mittaz-Crettol', \\ Ricardo Azambuja ${ }^{5}$, Lilian Okada ${ }^{5}$, Denise P. Cavalcanti6, Juliana Cuzzi 7 , \\ Mariângela Badalotti5, Rafaella Petracco5, Álvaro Petracco5, Lavinia Schüler-Faccini ${ }^{1,2}$, \\ Maria Teresa V. Sanseverino ${ }^{2 *}$ \\ ${ }^{1}$ Department of Genetics, Federal University of Rio Grande do Sul, Porto Alegre, Brazil \\ ${ }^{2}$ Medical Genetics Service, Hospital de Clínicas de Porto Alegre, Porto Alegre, Brazil \\ ${ }^{3}$ School of Medicine, Federal University of Rio Grande do Sul, Porto Alegre, Brazil \\ ${ }^{4}$ Division of Molecular Pediatrics, Lausanne University Hospital, Lausanne, Switzerland \\ ${ }^{5}$ Fertilitat Clínica de Reprodução, Porto Alegre, Brazil \\ ${ }^{6}$ Skeletal Dysplasia Group, Medical Genetics Department, Faculty of Medicine, State University of Campinas, \\ Campinas, Brazil \\ ${ }^{7}$ Genesis Genetics Diagnosis, São Paulo, Brazil \\ Email: ${ }^{*}$ msanseverino@hcpa.ufrgs.br
}

Received 19 March 2014; revised 15 April 2014; accepted 22 April 2014

Copyright (C) 2014 by authors and Scientific Research Publishing Inc.

This work is licensed under the Creative Commons Attribution International License (CC BY).

http://creativecommons.org/licenses/by/4.0/

(c) (7) Open Access

\section{Abstract}

Atelosteogenesis type II (A02) and diastrophic dysplasia (DTD) are two recessively inherited, severe skeletal dysplasias caused by mutations in the $S L C 26 A 2$ gene. AO2 is an invariably lethal condition, while DTD patients may reach adult life, although both diseases have overlapping diagnostic features. Here we report a patient with an intermediate phenotype between AO2 and DTD and present the successful application of preimplantation genetic diagnosis (PGD) in this situation. Sequencing of SLC26A2 alleles in the infant identified two compound heterozygous mutations, p.Arg178Ter and p.Arg279Trp, of paternal and maternal origin, respectively. At request from the parents, PGD was developed by haplotype mapping of parental $S L C 26 A 2$ alleles in eleven five-day embryos. Transference to the mother was attempted twice, finally resulting in pregnancy and delivery of a healthy baby. This exemplifies the utility of PGD for inherited lethal conditions with a significant risk of recurrence, and highlights the importance of accurate diagnosis of skeletal dys-

${ }^{*}$ Corresponding author.

How to cite this paper: Mattos, E.P., et al. (2014) Atelosteogenesis Type 2/Diastrophic Dysplasia Phenotypic Spectrum: From Prenatal to Preimplantation Genetic Diagnosis. Open Journal of Obstetrics and Gynecology, 4, 399-404. 
plasias with prenatal manifestation.

Keywords Atelosteogenesis Type 2, Diastrophic Dysplasia, Preimplantation Genetic Diagnosis, Prenatal
Diagnosis, Skeletal Dysplasia

\section{Introduction}

Skeletal dysplasias, or osteochondrodysplasias (OCDs), comprise a vast group of genetic diseases affecting the formation of the skeleton [1]. About $40 \%$ of OCDs can be recognized in the prenatal period [2], and combined; OCDs are frequent malformations that significantly contribute to perinatal death [3]. An interesting group of recessive OCDs is due to mutations in the diastrophic dysplasia sulfate transporter gene (DTDST), also known as SLC26A2. Conditions with this genetic basis range from severe achondrogenesis type 1B (ACG1B) and atelosteogenesis type II (AO2), to diastrophic dysplasia (DTD), and milder recessively inherited multiple epiphyseal dysplasia (rMED) and diastrophic dysplasia variant (mDTD) [4]-[6]. ACG1B and AO2 are lethal conditions [7], while individuals with DTD and rMED reach adult life [8].

AO2 comprises micromelia with adducted ("hitchhiker") thumbs and talipes equinovarus with a wide gap between the first and second toes. Micrognathia, cleft palate, and small chest can also be present [9]. Shortening of tubular bones, humeri with U- or V-shaped distal ends, bowed radiuses and ulnae, and unossified pubic bones are also observed [9]. DTD usually has a similar phenotype, which may hinder the antenatal distinction between AO2 and severe DTD [8] [9]. This points to a transitional phenotype between these conditions, in which different SLC26A2 alleles contribute to a spectrum of OCDs [10] [11].

We present a Brazilian fetus with a severe OCD diagnosed as an intermediate phenotype between AO2 and severe DTD by post mortem radiological investigation, the molecular diagnosis and subsequent preimplantation genetic diagnosis (PGD) offered to the family, leading to pregnancy and delivery of a healthy infant.

\section{Methods}

\subsection{Detection of Mutations}

The SLC26A2 gene was amplified by PCR and screened for the four most common SLC26A2 mutations by restriction enzyme digestion and gel electrophoresis with positive and negative controls. Subsequently, selective fragments of the gene were analyzed by bidirectional fluorescent direct sequencing. Results have been confirmed in a second amplification product. Primer sequences and restriction enzymes used are listed on Table 1.

\subsection{Preimplantation Genetic Diagnosis}

The established protocol followed the guidelines of the Preimplantation Genetic Diagnosis (PGD) International

Table 1. Polymersase chain reaction primers and restriction enzymes used for SLC26A2 screening.

\begin{tabular}{|c|c|c|c|}
\hline \multirow{2}{*}{ SLC26A2 utation ${ }^{a}$} & \multicolumn{3}{|c|}{ Reagents } \\
\hline & Forward primer & Reverse primer & $\begin{array}{c}\text { Restriction } \\
\text { enzyme }\end{array}$ \\
\hline c. $-26+2 \mathrm{~T}>\mathrm{C}$ & $\begin{array}{l}\text { 5’-CTTCGGAGTC } \\
\text { CGAGCGATGG-3' }\end{array}$ & $\begin{array}{l}\text { 5’-GACCCCTGAT } \\
\text { CTGGGATTCT-3' }\end{array}$ & Hph I \\
\hline c.559 C > T & $\begin{array}{l}\text { 5’-AGGAAGCTGA } \\
\text { ACCATCTATC-3' }\end{array}$ & $\begin{array}{l}\text { 5’-GATTCCTCAG } \\
\text { ATCCCTTAGAG-3' }\end{array}$ & Dde I \\
\hline c. $862 \mathrm{C}>\mathrm{T}$ & $\begin{array}{l}\text { 5’-CTCCATGCAA } \\
\text { GAAATGTCAGG-3' }\end{array}$ & $\begin{array}{l}\text { 5’-TGATACAGTG } \\
\text { ATAGCAAAACC-3' }\end{array}$ & Sty I \\
\hline c. $1984 \mathrm{~T}>\mathrm{A}$ & $\begin{array}{c}\text { 5'-CAACCCAATC } \\
\text { TTAATAAAGGTG-3' }\end{array}$ & $\begin{array}{l}\text { 5’-CTAGACATTC } \\
\text { TTCTATCTACC-3’ }\end{array}$ & - \\
\hline
\end{tabular}

a. Sequencing primer: 5'-CAACCCAATCTTAATAAAGGTG-3'. 
Society [12]. Twenty-four oocytes were fertilized and 19 embryos were successfully produced. DNA was extracted from trophectoderm cells of eleven viable embryos on the fifth day post-fertilization. All biopsied embryos were cryopreserved until transference to the mother. Whole-genome amplification was performed and samples were screened for SLC26A2 alleles by parental haplotype analysis, based on the mutations identified. As requested by the parents, 24-chromosome array-comparative genomic hybridization (aCGH) was also employed for detection of chromosomal abnormalities.

\subsection{Ethical Approval}

The individuals reported here were informed and consented with the anonymous disclosure of their medical information. This work was ethically reviewed and approved by the Institutional Review Board of Hospital de Clínicas de Porto Alegre.

\section{Results}

Recently, a non-consanguineous couple (32-year-old father and 30-year-old mother) of Portuguese ancestry was referred to our Institution during their second pregnancy due to recurrence of detection of a severe OCD. Their previous pregnancy ended at 16 weeks of gestation, after short fetal long bones and trunk constriction were detected. During the second pregnancy, ultrasonography identified again very short long bones and pregnancy ended at 17 weeks. Postnatal physical examination revealed a female fetus with severely shortened limbs and fingers with hitchhiker thumbs, varus deformity of lower limbs, talipes equinovarus with a wide gap between first and second toes, relative macrocephaly, midface hypoplasia, micrognathia, and short trunk (Figure 1(a)). Babygram showed narrowing of upper thorax, thin ribs, round ilia, and short long bones and metacarpals (Figure 1(b)). These findings were very suggestive of either AO2 or DTD.

Sequencing of the mother-father-child trio characterized the fetus as a compound heterozygote for two known pathogenic SLC26A2 point mutations, c.559C > T (p.Arg178Ter) of paternal, and c.862 C > T (p.Arg279Trp) of maternal origins (Figure 1(c) and Figure 2). This genotype has been previously observed and associated with AO2 and DTD [9], and the 17-week fetus presented indeed characteristic radiographic findings of both disorders.

A PGD protocol was elected by the family for a subsequent pregnancy. Upon the establishment of a PGD protocol, haplotype reconstructions identified only two embryos carrying both wild type SLC26A2 alleles (Table 2). These were firstly transferred to the mother, but no pregnancy developed. Of the other 9 samples, seven carried just one of the two pathogenic alleles, and only five were chromosomally balanced. The parents opted for the implantation of two heterozygous carrier embryos, and this second attempt resulted in pregnancy and delivery of a healthy infant, although the baby was not postnatally screened for SLC26A2 mutations.

\section{Discussion}

In the present study, the evaluated fetus had clinical findings compatible with both AO2 and severe DTD. In fact, the skeletal abnormalities reported here are strikingly similar to those identified in patients reported by MacíasGomez and colleagues (2004) [10] and Maeda and colleagues [11], who were also diagnosed with an intermediate AO2/DTD condition.

In SLC26A2, the p.Arg279Trp mutation impairs protein activity by approximately 50\%, when compared to the wild-type transporter [13]. Additionally, p.Arg178Ter is the second most common mutation found in patients of the DTDST spectrum [4]. In Xenopus laevis oocytes, it was shown that p.Arg178Ter behaves almost as a null allele, practically abolishing the protein's sulfate transport activity [14].

The substitution p.Arg279Trp has been found in AO2 mostly in compound heterozygosity with p.Arg178Ter or with another null allele [7] [10] [13] [15], and the p.Arg178Ter + p.Arg279Trp genotype has been identified in almost half of the patients with AO2, as well as in $20 \%$ of patients with DTD diagnosed at the Lausanne Molecular Pediatrics laboratory (L. Mittaz-Crettol, personal communication). Barbosa and colleagues (2011) identified compound heterozygosity for p.Arg178Ter and p.Arg279Trp in seven Portuguese patients diagnosed with classical DTD, and commented on the phenotypic overlap due to SLC26A2 mutations [8]. Another group reported both mutations in an infant with clinical and skeletal findings suggestive of a severe variant of DTD or a mild form of AO2 [10]. These observations reinforce the notion that the same SLC26A2 mutations may not 


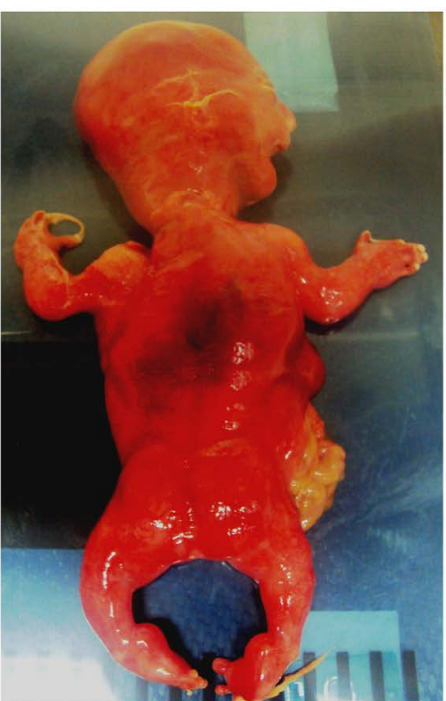

(a)

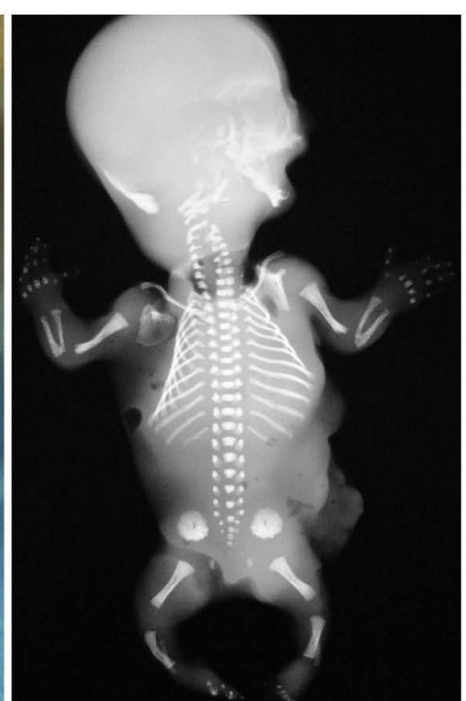

(b)

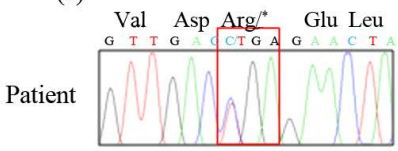

Leu Pro Arg/Trp Thr Asn
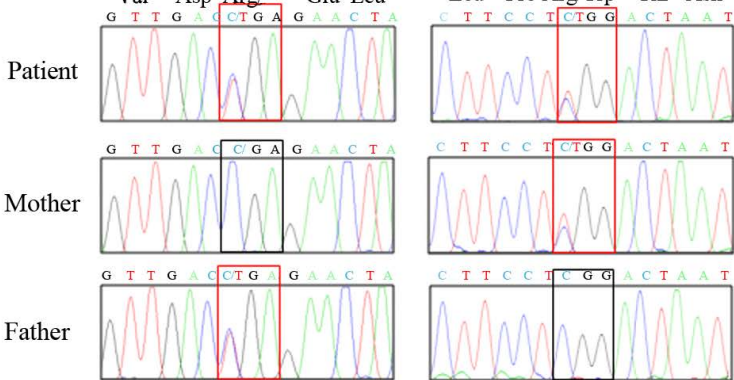

c. $[559 \mathrm{C}>\mathrm{T}] ;$ p. $\left[\operatorname{Arg} 178^{*}\right]$
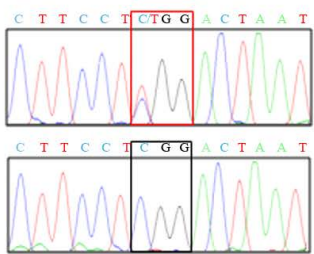

c.[862C > T]; p.[Arg279Trp]

(c)

Figure 1. Phenotype of the fetus at 17 weeks of gestation. A: Fetus appearance upon necropsy. Note the short trunk and limbs and the bowing of lower limbs with club feet. Hands with the characteristic "hitchhiker" thumb and feet with a wide gap between the first and second toes are also present. B: Whole-body radiography of the fetus, revealing short long bones, thoracic constriction, thin ribs, and round ilia with unossified pubis. C: $S L C 26 A 2$ sequencing of the mother-father-child trio. Electropherograms depict heterozygous peaks corresponding to the c.559 $\mathrm{C}>\mathrm{T}$ substitution (p.Arg178Ter) of paternal origin, and to the c.882 C > T substitution (p.Arg279Trp) of maternal origin.
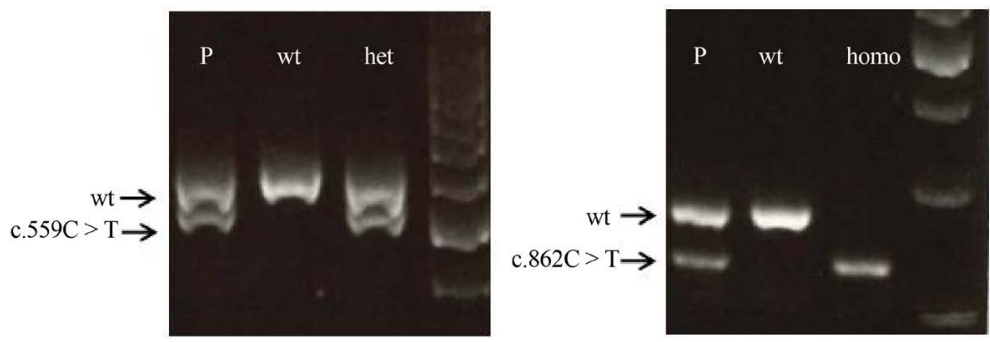

Figure 2. Restriction enzyme digestion of PCR products amplified from the DNA of the fetus. Top legend: P stands for patient, wt for a wild type control, het for a heterozygous control sample and homo for a homozygous control sample for the given mutation. Side legend: wt stands for the non-digested allele. The allele being digested is presented with the description of the mutation that gives rise to the new restriction site. 
Table 2. Preimplantation genetic diagnosis for AO2/DTD and embryo selection.

\begin{tabular}{|c|c|c|c|c|}
\hline \multirow{2}{*}{ Sample } & \multicolumn{4}{|c|}{ Embryo characteristics } \\
\hline & Inferred alleles $^{a}$ & aCGH result & Interpretation & Fate of embryo \\
\hline 2 & p.Arg178Ter wt ${ }^{\mathrm{c}}$ & $45, X X ;-2$ & $\begin{array}{l}\text { Heterozygous carrier (paternal mutant } \\
\text { allele); chromosome } 2 \text { monosomy }\end{array}$ & Not used \\
\hline 5 & p.Arg178Ter/wt & $45, X X ;-17$ & $\begin{array}{l}\text { Heterozygous carrier (paternal mutant } \\
\text { allele); chromosome } 17 \text { monosomy }\end{array}$ & Not used \\
\hline 6 & wt/p.Arg279Trp & 46, XY & $\begin{array}{l}\text { Heterozygous carrier } \\
\text { (maternal mutant allele) }\end{array}$ & $\begin{array}{c}\text { Transferred attempt \#2, } \\
\text { healthy infant born }\end{array}$ \\
\hline 7 & p.Arg178Ter/wt & $46, \mathrm{XX}$ & $\begin{array}{l}\text { Heterozygous carrier } \\
\text { (paternal mutant allele) }\end{array}$ & Transferred attempt \#2 \\
\hline 9 & wt/p.Arg279Trp & 46, XY & $\begin{array}{l}\text { Heterozygous carrier } \\
\text { (maternal mutant allele) }\end{array}$ & Cryopreserved \\
\hline 10 & $\mathrm{wt} / \mathrm{wt}$ & 46, XX & Wild type at the SLC26A2 locus & Transferred attempt \#1 \\
\hline 11 & wt/p.Arg279Trp & 46, XY & $\begin{array}{l}\text { Heterozygous carrier } \\
\text { (maternal mutant allele) }\end{array}$ & Cryopreserved \\
\hline 13 & wt/p.Arg279Trp & $46, \mathrm{XX}$ & $\begin{array}{l}\text { Heterozygous carrier } \\
\text { (maternal mutant allele) }\end{array}$ & Cryopreserved \\
\hline 14 & $\begin{array}{l}\text { p.Arg178Ter/ } \\
\text { p.Arg279Trp }\end{array}$ & $\mathrm{NT}^{\mathbf{b}}$ & $\begin{array}{c}\text { Compound heterozygous mutant } \\
\text { (maternal and paternal mutant alleles) }\end{array}$ & Not used \\
\hline 17 & $\begin{array}{l}\text { p.Arg178Ter/ } \\
\text { p.Arg279Trp }\end{array}$ & NT & $\begin{array}{c}\text { Compound heterozygous mutant } \\
\text { (maternal and paternal mutant alleles) }\end{array}$ & Not used \\
\hline 19 & wt/wt & 46, XY & Wild type at the SLC26A2 locus & Transferred attempt \#1 \\
\hline
\end{tabular}

always translate into a single, characteristic phenotype, implying genetic and/or environmental factors influencing sulfate transport activity and clinical outcome.

\section{Conclusion}

This report exemplifies the growing application of PGD for severe monogenic diseases [16]. To the best of our knowledge, this is one of the first published descriptions on the application of PGD for a recessive lethal skeletal dysplasia with phenotypic characteristics between AO2 and DTD, leading to the successful live birth of a healthy infant. This exemplifies a growing trend in the medical genetics field, especially for the management of lethal recurrent Mendelian traits with substantial risk of recurrence.

\section{Acknowledgements}

The authors would like to thank the subjects reported here and Hospital de Clínicas de Porto Alegre Research Fund 12-0467 for supporting this publication.

\section{References}

[1] Warman, M.L., Cormier-Daire, V., Hall, C., Krakow, D., Lachman, R., LeMerrer, M., et al. (2011) Nosology and Classification of Genetic Skeletal Disorders: 2010 Revision. American Journal of Medical Genetics Part A, 155A, 943-968. http://dx.doi.org/10.1002/ajmg.a.33909

[2] Krakow, D., Alanay, Y., Rimoin, L.P., Lin, V., Wilcox, W.R., Lachman, R.S. and Rimoin, D.L. (2008) Evaluation of Prenatal-Onset Osteochondrodysplasias by Ultrasonography: A Retrospective and Prospective Analysis. American Journal of Medical Genetics Part A, 146A, 1917-1924. http://dx.doi.org/10.1002/ajmg.a.32269

[3] Barbosa-Buck, C.O., Orioli, I.M., da Graça Dutra, M., Lopez-Camelo, J., Castilla, E.E. and Cavalcanti, D.P. (2012) Clinical Epidemiology of Skeletal Dysplasias in South America. American Journal of Medical Genetics Part A, 158A, 1038-1045. http://dx.doi.org/10.1002/ajmg.a.35246

[4] Rossi, A. and Superti-Furga, A. (2001) Mutations in the Diastrophic Dysplasia Sulfate Transporter (DTDST) Gene (SLC26A2): 22 Novel Mutations, Mutation Review, Associated Skeletal Phenotypes, and Diagnostic Relevance. 
Human Mutation, 17, 159-171. http://dx.doi.org/10.1002/humu.1

[5] Rossi, A., Kaitila, I., Wilcox, W.R., Rimoin, D.L., Steinmann, B., Cetta, G. and Superti-Furga, A. (1998) Proteoglycan Sulfation in Cartilage and Cell Cultures from Patients with Sulfate Transporter Chondrodysplasias: Relationship to Clinical Severity and Indications on the Role of Intracellular Sulfate Production. Matrix Biology, 17, 361-369. http://dx.doi.org/10.1016/S0945-053X(98)90088-9

[6] Panzer, K.M., Lachman, R., Modaff, P. and Pauli, R.M. (2008) A Phenotype Intermediate between Desbuquois Dysplasia and Diastrophic Dysplasia Secondary to Mutations in DTDST. American Journal of Medical Genetics Part A, 146A, 2920-2924. http://dx.doi.org/10.1002/ajmg.a.32543

[7] Hästbacka, J., Superti-Furga, A, Wilcox, W.R., Rimoin, D.L., Cohn, D.H. and Lander, E.S. (1996) Atelosteogenesis Type II Is Caused by Mutations in the Diastrophic Dysplasia Sulfate-Transporter Gene (DTDST): Evidence for a Phenotypic Series Involving Three Chondrodysplasias. American Journal of Human Genetics, 58, 255-262.

[8] Barbosa, M., Sousa, A.B., Medeira, A., Lourenço, T., Saraiva, J., Pinto-Basto, J., et al. (2011) Clinical and Molecular Characterization of Diastrophic Dysplasia in the Portuguese Population. Clinical Genetics, 80, 550-557. http://dx.doi.org/10.1111/j.1399-0004.2010.01595.x

[9] Bonafé, L., Mittaz-Crettol, L., Ballhausen, D. and Superti-Furga, A. (2014) Atelosteogenesys Type 2. In: Pagon, R.A.,

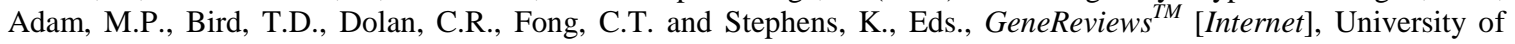
Washington, Seattle. Retrieved from http://www.ncbi.nlm.nih.gov/books/NBK1317/

[10] Macías-Gómez, N.M., Mégarbané, A., Leal-Ugarte, E., Rodríguez-Rojas, L.X. and Barros-Núñez, P. (2004) Diastrophic Dysplasia and Atelosteogenesis Type II as Expression of Compound Heterozygosis: First Report of a Mexican Patient and Genotype-Phenotype Correlation. American Journal of Medical Genetics Part A, 129A, 190-192. http://dx.doi.org/10.1002/ajmg.a.30149

[11] Maeda, K., Miyamoto, Y., Sawai, H., Karniski, L.P., Nakashima, E., Nishimura, G. and Ikegawa, S. (2006) A Compound Heterozygote Harboring Novel and Recurrent DTDST Mutations with Intermediate Phenotype between Atelosteogenesis Type II and Diastrophic Dysplasia. American Journal of Medical Genetics Part A, 140, 1143-1147. http://dx.doi.org/10.1002/ajmg.a.31225

[12] Preimplantation Genetic Diagnosis International Society (2008) Guidelines for Good Practice in PGD: Programme Requirements and Laboratory Quality Assurance. Reproductive Biomedicine Online, 16, 134-147. http://dx.doi.org/10.1016/S1472-6483(10)60567-6

[13] Karniski, L.P. (2004) Functional Expression and Cellular Distribution of Diastrophic Dysplasia Sulfate Transporter (DTDST) Gene Mutations in HEK Cells. Human Molecular Genetics, 13, 2165-2171. http://dx.doi.org/10.1093/hmg/ddh242

[14] Karniski, L.P. (2001) Mutations in the Diastrophic Dysplasia Sulfate Transporter (DTDST) Gene: Correlation between Sulfate Transport Activity and Chondrodysplasia Phenotype. Human Molecular Genetics, 10, 1485-1490. http://dx.doi.org/10.1093/hmg/10.14.1485

[15] Rossi, A., van der Harten, H.J., Beemer, F.A., Kleijer, W.J., Gitzelmann, R., Steinmann, B. and Superti-Furga, A. (1996) Phenotypic and Genotypic Overlap between Atelosteogenesis Type 2 and Diastrophic Dysplasia. Human Genetics, 98, 657-661. http://dx.doi.org/10.1007/s004390050279

[16] Harper, J.C., Wilton, L., Traeger-Synodinos, J., Goossens, V., Moutou, C., SenGupta, S.B., et al. (2012) The ESHRE PGD Consortium: 10 Years of Data Collection. Human Reproduction Update, 18, 234-247. http://dx.doi.org/10.1093/humupd/dmr052 